\title{
Computational Screening of Phytochemicals from Medicinal plants as COVID-19 Inhibitors
}

Khandelwal Alisha ${ }^{1}$, Sharma Tripti ${ }^{1}$

${ }^{1}$ School of Pharmaceutical Sciences, Siksha 'O' Anusandhan Deemed to be University, Bhubaneswar - 751003, Odisha, India Email: triptisharma@soa.ac.in

Address for Correspondence:

Dr.Tripti Sharma, School of Pharmaceutical Sciences, Siksha 'O' Anusandhan Deemed to be University, Campus - 2, Kalinga Nagar, Bhubaneswar 751003, Odisha, India. Email ID: triptisharma@soa.ac.in 


\begin{abstract}
Aggressive strategies are planned globally to combat the newly developed COVID-19 worldwide. This pandemic virus has spread and affected globally leading to an increase in death tolls. Currently, no effective drug for treatment and management of the disease is available. Nature has gifted us with valuable resources in the form of medicinal plants which are used since time immemorial for the treatment of various diseases. In this research a dataset of plant based bioactive compound was developed. A total of 101 phytochemicals were selected, virtually designed and its binding affinity with ACE enzyme was studied by molecular docking. Human ACE related carboxypeptidase and complex (PDB ID: 1R42) and (PDB ID: 6CS2) were selected for molecular docking studies as corona virus binds to ACE2 to enter into the host cell. Docking score results revealed that almost all selected phytochemicals binds to the pocket of the human ACE protein with high binding affinity and the scores were compared with chloroquine and hydroxychloroquine. The drug likeliness and ADMET analysis of all the screened compounds were performed. Two potential compound 6 - $\alpha$-acetoxygedunin and echitamine exhibited optimum binding with both the receptor.These phytochemicals can serve as lead molecule for further optimization and drug development against COVID-19. Therefore, it is predicted that the insights in the present study could be regarded valuable towards development of natural inhibitor of this virus.
\end{abstract}

Keywords: Molecular docking, phytochemicals, COVID-19, 6- $\alpha$-acetoxygedunin, echitamine. 


\section{INTRODUCTION}

The early episodes the novel coronavirus evolved in Wuhan, China, in December 2019 and later engulfed the whole world [1]. The World Health Organization announced the flare-up to be a public health emergency of international concern on 30 January 2020[2]. The COVID-19 is a severe acute respiratory syndrome coronavirus 2 (SARS-CoV-2) which causes mild to severe respiratory tract infections [3].Although there is recovery of COVID-19 positive patients but still there is no authenticated medication to inhibit/kill this deadly virus. The disease is highly contagious and the longest watched span of viral shedding was for 37 days [4]. Patients suffered from multiple disorders with hypertension being the most widely recognized trailed by diabetes, heart problems and even multiple organ failure. SARS-CoV-2 is reported to utilise ACE-2 for entry into the target cells [5]. ACE 2 , a protective protein widely distributed in human body is down regulated after viral infection, this decreases the degradation of angiotensin II and reduces production of angiotensin (1-7). Imbalance of these proteins in the RAS cascade leads to target organ damage [6].

The phytochemical from medicinal plants are used in the treatment of various diseases since ancient times. As compared to the synthetic drug(s) plant derived antiviral agents are associated with lesser side effects. Thus, phytochemicals may be a better alternative for the treatment of this dreaded pandemic disease. Bioactive phytochemicals like polyphenols, alkaloids, coumarins, saponins, flavonoids, terpenoids, limonoids, steroids, polysaccharides are found to inhibit genetically and functionally diverse viruses [7-9].

In this situation of great crisis due to the spread of the pandemic search for a drug to combat COVID19 is need of the hour. In-silico techniques are inexpensive, fast and reliable methods in initial drug discovery and developments process. The objective of this study is to analyse the inhibitory action of bioactive molecules from medicinal plants on ACE-2 proteins by computational docking studies.

\section{MATERIALS AND METHODS}

\subsection{Retrieval of Phytochemical ligands}

A series of 3-D structures of different phytoconstituents obtained from phytochemical databases were virtually retrieved from NCBI Pubchem (https://pubchem.ncbi.nlm.nih.gov ) in structure-data file (SDF) format and drawn by using Marvin Sketch and saved in .mol format in mol file. The mol file of the ligands was converted to PDBQT format using virtual autodocking software tool PyRx to obtain best atomic conformation of the ligands [10].

\subsection{Retrieval of Protein}

The 3-D X-ray crystalline structure of the two human ACE related protein with PDB ID:1R42; native human angiotensin converting enzyme-related carboxypeptidase (ACE-2)and PDB ID:6CS2;SARS 
spike glycoprotein - human ACE-2 complex were obtained from RCSB protein data bank (https://www.rcsb.org/structure/1R42) and (https://www.rcsb.org/structure/6CS2) at atomic resolution $2.2 \AA$ and $4.4 \AA$ respectively (Figure 1). The water, unwanted residues and chains were removed from the proteins using Notepad ++ 7.8.6 and further repaired using WHATIF server and saved in PDB format[11]. The 3-D ligplot graphs and 2-D interaction of the ligands and protein were generated using Discovery Studio 4.5. The binding site of the proteins was analysed using CASTp web server [12].
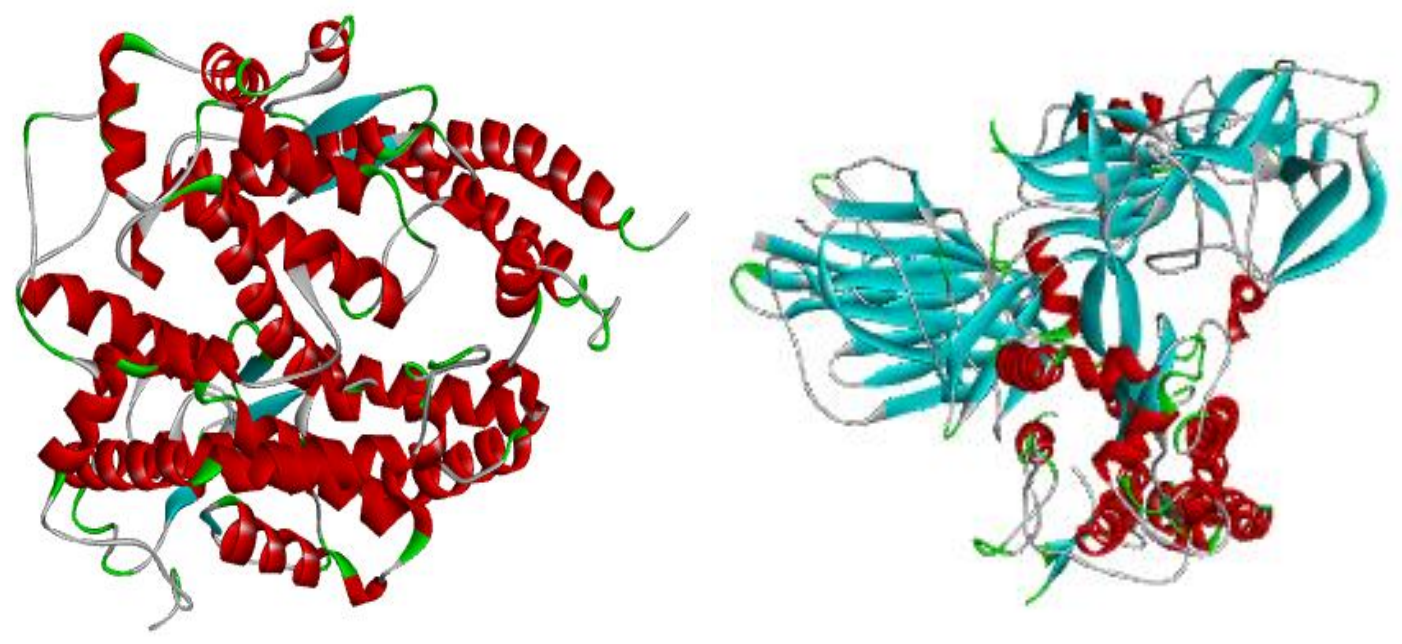

Figure 1. Native human ACE-2-related carboxypeptidase (PDB ID: 1R42) and SARS spike glycoprotein - human ACE-2 complex (PDB ID: 6CS2)

\subsection{Molecular Docking}

The molecular docking of the active site of the ACE-2 SARS protein with the selected series of phytochemicals was carried out in Autodock virtual docking software PyRx [13]. The ligands were retrieved in PyRx and protein 1R42 and 6CS2 were loaded in PDB format and converted in PDBQT format by water removal, hydrogen atom and kollman charges addition. The grid centre was positioned on the active site of the protein $1 \mathrm{R} 42$ and $6 \mathrm{CS} 2$. The grid value of $1 \mathrm{R} 42$ and $6 \mathrm{CS} 2$ for autogrid calculation were positioned at $\mathrm{X}=53.79, \mathrm{Y}=60.55, \mathrm{Z}=30.87$ and $\mathrm{X}=161.014, \mathrm{Y}=195.19, \mathrm{Z}=190.43$ respectively. The best binding affinity was selected from a set containing nine interacting poses after selection of the ligand and proteins in PDBQT format and running them in vina wizard simultaneously. The docking score of the virtually prepared phytochemicals with protein were compared with chloroquine and hydrochloroquine. The binding interactions of protein with selected candidate and type of interacting bond were analysed using Discovery Studio 4.5.

\subsection{Drug-likeness and ADMET Prediction}


The Lipinski rule of five signifies the drugability of the compound. This was calculated using Molinspiration tool https://www.molinspiration.com. The molecular weight, no of hydrogen bond donor and acceptor, no of Lipinski violations, no of rotatable bonds all were calculated. The ADMET calculations were performed using pkCSM [14] which produces the results based on graph-based signatures. Intestinal absorption, volume of distribution, blood brain barrier permeability, total clearance, LD 50 and mutagenicity were evaluated.

\section{RESULTS AND DISCUSSIONS}

Phytochemicals provide a strong root to the growing commercialization of medicines. Their biggest advantage is that they are nontoxic and rescue the body from any ailment with less or even no side effect. The use of phytochemicals for treatment of different diseases is an ancient concept. Many researches have proved that phytochemicals are less or even non-toxic and has been used to treat even life-threatening diseases [15]. A total of 101 phytochemicals belonging to various classes like alkaloids, glycosides, flavanoids, flavagline terpenes, terpenoids, lignan, tannins, phenols, coumarin, polysaccharides, resinoids and fatty acids were selected from databases and docked with human ACE2 SARS protein.

\subsection{Physicochemical and drug-likenessprediction}

All the selected phytochemicals were virtually screened against Lipinski rule (RO5) using Molinspiration tool. The TPSA, no of hydrogen donor and acceptor, $\log$ P, molecular weight (MW), no of atoms and rotatable bonds for almost every candidate were within limits. The physicochemical derivative parameters are presented in Table 1.

Table 1.Physicochemical properties of selected phytochemical

\begin{tabular}{|c|c|l|c|c|c|c|c|c|c|}
\hline Sr no. & Compounds & $\mathbf{l o g p}$ & $\mathbf{T P S A}$ & $\mathbf{n}$ & $\begin{array}{c}\mathbf{M W} \\
(\mathbf{5 0 0})\end{array}$ & $\begin{array}{c}\text { nON } \\
(\mathbf{1 0})\end{array}$ & $\begin{array}{c}\text { nOHNH } \\
(\mathbf{5 5})\end{array}$ & $\mathbf{n V}$ & nrotb \\
\hline 1 & Thalimonine & 2.77 & 49.41 & 27 & 369.42 & 6 & 0 & 0 & 3 \\
\hline 2 & Indole & 2.16 & 15.79 & 9 & 117.15 & 1 & 1 & 0 & 0 \\
\hline 3 & Cephaeline & 3.33 & 63.20 & 34 & 466.62 & 6 & 2 & 0 & 6 \\
\hline 4 & Emetine & 3.64 & 52.20 & 35 & 480.65 & 6 & 1 & 0 & 7 \\
\hline 5 & Psychotrine & 4.40 & 63.53 & 34 & 464.61 & 6 & 1 & 0 & 6 \\
\hline 6 & Alangine & 2.39 & 52.93 & 22 & 303.40 & 4 & 2 & 0 & 4 \\
\hline 7 & Tubulosine & 4.86 & 69.75 & 35 & 475.63 & 6 & 3 & 0 & 5 \\
\hline 8 & Isotubulosine & 4.86 & 69.75 & 35 & 475.63 & 6 & 3 & 0 & 5 \\
\hline 9 & Conessine & 4.79 & 6.48 & 26 & 356.60 & 2 & 0 & 0 & 1 \\
\hline 10 & Deoxytubulosine & 5.36 & 49.52 & 34 & 459.63 & 5 & 2 & 1 & 5 \\
\hline 11 & Ankorine & 2.84 & 62.16 & 24 & 335.44 & 5 & 2 & 0 & 5 \\
\hline 12 & Conessidine & 4.38 & 24.39 & 24 & 326.53 & 2 & 1 & 0 & 1 \\
\hline 13 & Quinazoline & 1.54 & 25.78 & 10 & 130.15 & 2 & 0 & 0 & 0 \\
\hline 14 & Conimine & 4.29 & 24.05 & 24 & 328.54 & 2 & 2 & 0 & 1 \\
\hline 15 & Isoconessimine & 4.54 & 15.27 & 25 & 342.57 & 2 & 1 & 0 & 1 \\
\hline 16 & Kurchessine & 5.47 & 6.48 & 27 & 372.64 & 2 & 0 & 1 & 3 \\
\hline
\end{tabular}




\begin{tabular}{|c|c|c|c|c|c|c|c|c|c|}
\hline 17 & Conessimine & 4.54 & 15.27 & 25 & 342.57 & 2 & 1 & 0 & 1 \\
\hline 18 & Alamarine & 1.21 & 84.59 & 25 & 338.36 & 6 & 2 & 0 & 2 \\
\hline 19 & holarrhimine & 1.98 & 72.27 & 24 & 332.53 & 3 & 5 & 0 & 2 \\
\hline 20 & Senoterpine & 0.53 & 33.12 & 11 & 149.19 & 2 & 1 & 0 & 0 \\
\hline 21 & Salsoline & 1.36 & 41.49 & 14 & 193.25 & 3 & 2 & 0 & 1 \\
\hline 22 & $\begin{array}{l}\text { 9-demethylprotoe } \\
\text { metinol }\end{array}$ & 2.62 & 52.93 & 22 & 305.42 & 4 & 2 & 0 & 4 \\
\hline 23 & Bharatamine & 2.62 & 32.70 & 21 & 281.36 & 3 & 1 & 0 & 1 \\
\hline 24 & Holafebrine & 3.65 & 46.25 & 23 & 317.52 & 2 & 3 & 0 & 1 \\
\hline 25 & Aristololactum & 3.73 & 60.56 & 22 & 293.28 & 5 & 1 & 0 & 1 \\
\hline 26 & Aristolic acid & 3.68 & 65.00 & 22 & 296.28 & 5 & 1 & 0 & 2 \\
\hline 27 & Echitamine & -2.27 & 78.79 & 28 & 385.48 & 6 & 3 & 0 & 3 \\
\hline 28 & Picrinine & 3.09 & 50.80 & 25 & 338.41 & 5 & 1 & 0 & 2 \\
\hline 29 & Akuammidine & 2.84 & 65.56 & 26 & 352.43 & 5 & 2 & 0 & 3 \\
\hline 30 & Strictamine & 3.38 & 41.91 & 24 & 322.41 & 4 & 0 & 0 & 2 \\
\hline 31 & Tetrahydroalstonine & 3.41 & 54.57 & 26 & 352.43 & 5 & 1 & 0 & 2 \\
\hline 32 & Quinine & 3.06 & 45.59 & 24 & 324.42 & 4 & 1 & 0 & 4 \\
\hline 33 & Cinchonidine & 3.03 & 36.36 & 22 & 294.40 & 3 & 1 & 0 & 3 \\
\hline 34 & Dihydroconessine & 4.97 & 6.48 & 26 & 358.61 & 2 & 0 & 0 & 1 \\
\hline 35 & Quercetin & 1.68 & 131.35 & 22 & 302.24 & 7 & 5 & 0 & 1 \\
\hline 36 & Baicalin & 0.55 & 187.12 & 32 & 446.36 & 11 & 6 & 2 & 4 \\
\hline 37 & Xanthohumol & 4.80 & 86.99 & 26 & 354.40 & 5 & 3 & 0 & 6 \\
\hline 38 & Taxifolin & 0.71 & 127.44 & 22 & 304.25 & 7 & 5 & 0 & 1 \\
\hline 39 & $\begin{array}{l}\text { Epigallocatechin 3- } \\
\text { gallate }\end{array}$ & 2.25 & 197.36 & 33 & 458.38 & 11 & 8 & 2 & 4 \\
\hline 40 & Glucuronide & 3.97 & 133.52 & 34 & 476.57 & 8 & 4 & 0 & 8 \\
\hline 41 & Ginkgetin & 5.97 & 159.80 & 42 & 566.52 & 10 & 4 & 2 & 5 \\
\hline 42 & $\begin{array}{c}\text { Tetrahydroxyflavano } \\
\text { ne }\end{array}$ & 0.80 & 107.22 & 21 & 288.25 & 6 & 4 & 0 & 1 \\
\hline 43 & Luteolin & 1.97 & 111.12 & 21 & 286.24 & 6 & 4 & 0 & 1 \\
\hline 44 & leucodelphinidin & 0.09 & 150.83 & 23 & 322.27 & 8 & 7 & 1 & 1 \\
\hline 45 & leucocyanidin & 0.38 & 130.60 & 22 & 306.27 & 7 & 6 & 1 & 1 \\
\hline 46 & $\begin{array}{c}\text { Decanoylphorb } \\
\text { ol-13 acetate }\end{array}$ & 5.88 & 130.37 & 40 & 560.73 & 8 & 3 & 2 & 13 \\
\hline 47 & Uvaol & 6.91 & 40.46 & 32 & 442.73 & 2 & 2 & 1 & 1 \\
\hline 48 & Ursolic acid & 6.79 & 57.53 & 33 & 456.71 & 3 & 2 & 1 & 1 \\
\hline 49 & Betulin & 7.16 & 40.46 & 32 & 442.73 & 2 & 2 & 1 & 2 \\
\hline 50 & Linalool & 3.21 & 20.23 & 11 & 154.25 & 1 & 1 & 0 & 4 \\
\hline 51 & Camphene & 3.33 & 0 & 10 & 136.24 & 0 & 0 & 0 & 0 \\
\hline 52 & P-Cymene & 3.90 & 0 & 10 & 134.22 & 0 & 0 & 0 & 1 \\
\hline 53 & 6- $\alpha$-Acetoxygedunin & 4.13 & 121.65 & 39 & 540.61 & 9 & 0 & 1 & 5 \\
\hline 54 & Honokiol & 5 & 40.46 & 20 & 266.34 & 2 & 2 & 1 & 5 \\
\hline 55 & SJP-L-5 & 3.47 & 57.24 & 24 & 329.35 & 6 & 0 & 0 & 5 \\
\hline 56 & Rhinacanthin E & 4.07 & 98.78 & 32 & 442.42 & 9 & 0 & 0 & 9 \\
\hline 57 & Rhinacanthin F & 3.95 & 98.78 & 32 & 444.44 & 9 & 0 & 0 & 10 \\
\hline 58 & Oleanane & 8.86 & 0.00 & 30 & 412.75 & 0 & 0 & 1 & 2 \\
\hline 59 & $\begin{array}{l}\text { Dammarenoic } \\
\text { acid }\end{array}$ & 8.08 & 57.53 & 33 & 458.73 & 3 & 2 & 1 & 8 \\
\hline 60 & Agastaquinone & 2.96 & 80.67 & 25 & 340.38 & 5 & 1 & 0 & 2 \\
\hline 61 & Saikosaponins & 1.98 & 207.99 & 55 & 780.99 & 13 & 8 & 3 & 6 \\
\hline 62 & Garciosaterpene A & 8.11 & 63.60 & 36 & 498.75 & 4 & 1 & 1 & 7 \\
\hline 63 & Garciosaterpene $\mathrm{C}$ & 7.23 & 54.37 & 33 & 454.69 & 3 & 1 & 1 & 5 \\
\hline
\end{tabular}




\begin{tabular}{|c|c|c|c|c|c|c|c|c|c|}
\hline 64 & Vaticinone & 5.93 & 34.14 & 31 & 424.67 & 2 & 0 & 1 & 4 \\
\hline 65 & Betulinic Acid & 7.04 & 57.53 & 33 & 456.71 & 3 & 2 & 1 & 2 \\
\hline 66 & Glycyrrhizin & 1.97 & 267.04 & 58 & 822.94 & 16 & 8 & 3 & 7 \\
\hline 67 & Betulinaldehyde & 7.62 & 37.30 & 32 & 440.71 & 2 & 1 & 1 & 2 \\
\hline 68 & Lupeol & 8.29 & 20.23 & 31 & 426.73 & 1 & 1 & 1 & 1 \\
\hline 69 & $\beta$-amyrin acetate & 8.55 & 26.30 & 34 & 468.77 & 2 & 0 & 1 & 2 \\
\hline 70 & Azulene & 3.17 & 0 & 10 & 128.17 & 0 & 0 & 0 & 0 \\
\hline 71 & Eucalyptol & 2.72 & 9.23 & 11 & 154.25 & 1 & 0 & 0 & 0 \\
\hline 72 & $\alpha$-Curcumene & 5.82 & 0 & 15 & 202.34 & 0 & 0 & 1 & 4 \\
\hline 73 & Elemol acetate & 5.06 & 26.30 & 19 & 264.41 & 2 & 0 & 1 & 5 \\
\hline 74 & $\beta$-Eudesmol & 4.01 & 20.23 & 16 & 222.37 & 1 & 1 & 0 & 1 \\
\hline 75 & Isololiolide & 1.84 & 46.53 & 14 & 196.25 & 3 & 1 & 0 & 0 \\
\hline 76 & $\alpha$-Spinasterol acetate & 8.45 & 26.30 & 33 & 454.74 & 2 & 0 & 1 & 7 \\
\hline 77 & Cycloeucalenol & 7.62 & 20.23 & 31 & 426.73 & 1 & 1 & 1 & 5 \\
\hline 78 & Cycloartenol & 8.21 & 20.23 & 31 & 426.73 & 1 & 1 & 1 & 4 \\
\hline 79 & Oleuropeic acid & 1.24 & 57.53 & 13 & 184.24 & 3 & 2 & 0 & 2 \\
\hline 80 & Madasiatic acid & 4.96 & 97.98 & 35 & 488.71 & 5 & 4 & 0 & 1 \\
\hline 81 & Asiaticoside & 0.37 & 315.21 & 67 & 959.13 & 19 & 12 & 3 & 10 \\
\hline 82 & Asiaticoside A & -0.55 & 335.44 & 68 & 975.13 & 20 & 13 & 3 & 10 \\
\hline 83 & Asiaticoside B & -0.61 & 335.44 & 68 & 975.13 & 20 & 13 & 3 & 10 \\
\hline 84 & Lupeol acetate & 8.71 & 26.30 & 34 & 468.77 & 2 & 0 & 1 & 3 \\
\hline 85 & $\alpha$-carotene & 9.79 & 0 & 40 & 536.89 & 0 & 0 & 2 & 10 \\
\hline 86 & SennosideA & 0.86 & 347.96 & 62 & 862.75 & 20 & 12 & 3 & 9 \\
\hline 87 & Silvestrol & 2.92 & 171.85 & 47 & 654.66 & 13 & 4 & 2 & 11 \\
\hline 88 & Loliolide & 1.84 & 46.53 & 14 & 196.25 & 3 & 1 & 0 & 0 \\
\hline 90 & Ellagic acid & 0.09 & 141.33 & 22 & 302.19 & 8 & 4 & 0 & 0 \\
\hline 91 & Calanolide A & 4.50 & 68.91 & 27 & 370.44 & 5 & 1 & 0 & 2 \\
\hline 92 & Hentriacontane & 10.2 & 0 & 31 & 436.85 & 0 & 0 & 1 & 28 \\
\hline 93 & Linoleic acid & 6.86 & 37.30 & 20 & 280.45 & 2 & 1 & 1 & 14 \\
\hline 94 & Oleic acid & 7.58 & 37.30 & 20 & 282.47 & 2 & 1 & 1 & 15 \\
\hline 95 & Palmitic acid & 7.06 & 37.30 & 18 & 256.43 & 2 & 1 & 1 & 14 \\
\hline 96 & Stearic acid & 8.07 & 37.30 & 20 & 284.48 & 2 & 1 & 1 & 16 \\
\hline 97 & Behenic acid & 9.13 & 37.30 & 24 & 340.59 & 2 & 1 & 1 & 20 \\
\hline 98 & Arachidic acid & 8.37 & 37.30 & 22 & 312.54 & 2 & 1 & 1 & 18 \\
\hline 99 & Chrysin & 2.94 & 70.67 & 19 & 254.24 & 4 & 2 & 0 & 1 \\
\hline 100 & Morin & 1.88 & 131.35 & 22 & 302.24 & 7 & 5 & 0 & 1 \\
\hline 101 & $\begin{array}{l}\alpha-D-G a l a c t u r o n i c \\
\text { acid }\end{array}$ & -2.77 & 127.44 & 13 & 194.14 & 7 & 5 & 0 & 1 \\
\hline 102 & Chloroquine & 5 & 28.16 & 22 & 319.88 & 3 & 1 & 1 & 8 \\
\hline 103 & Hydroxy chloroquine & 4 & 48.38 & 23 & 335.88 & 4 & 2 & 0 & 9 \\
\hline
\end{tabular}

Log P: Partition coefficient; TPSA: Total Polar Surface Area; n: No of atoms; MW: Molecular weight; noN: No of hydrogen bond acceptor; nOHNH: No of hydrogen bond donor; nV: No of Violation; nrotb: No of rotatable bond.

\subsection{Molecular docking}

We docked all the selected phytochemical from the database with 1R42 and 6CS2. The docking score obtained ranges from -4.2 to -13.4 and -3.1 to -11.8 with 1 R42 and 6CS2 respectively. Our study revealed that the best docking energy was exhibited by 6 - $\alpha$-acetoxygedunin with binding affinity of - 
$15.4 \mathrm{kcal} / \mathrm{mol}$ for $1 \mathrm{R} 42$ and $-13.1 \mathrm{kcal} / \mathrm{mol}$ for $6 \mathrm{CS} 2$ followed by echitamine $(-12.1 \mathrm{kcal} / \mathrm{mol}$ for $1 \mathrm{R} 42$ and $-10.3 \mathrm{kcal} / \mathrm{mol}$ for $6 \mathrm{CS} 2$ ). The 2-D structures of the phytochemicals and their docking score are enlisted in Table 2. 3-D binding and ligplot analysis of 6- $\alpha$-acetoxygedunin with 1R42 and 6CS2 was carried out in Discovery Studio to predict the interacting amino acid binding site depicted in Figure 2 and Figure 3. After molecular docking studies 6- $\alpha$-acetoxygedunin was found bonded to the amino acid through conventional $\mathrm{H}$ bond: Tyr127, Pi-cation: Phe 504 and vanderwaal interactions: His 505, Try271, Asn 149, Leu 144 of 1R42 and vanderwaal interactions: Thr 51, Leu 52, Lys 291, Ser 292, Phe 293 of 6 CS2.

Table 2. 2-D structure and docking score of the phytochemicals

\begin{tabular}{|c|c|c|c|c|c|c|}
\hline Class & $\begin{array}{l}\text { Sr } \\
\text { no. }\end{array}$ & Compounds & Structure & CID & 1R42 & 6CS2 \\
\hline \multirow{6}{*}{$\begin{array}{l}A \\
L \\
K \\
A \\
L \\
\text { O } \\
\text { I } \\
D \\
S\end{array}$} & 1 & Thalimonine & & 10893946 & -6.5 & -6.1 \\
\hline & 2 & Indole & & 798 & -4.4 & -3.9 \\
\hline & 3 & Cephaeline & & 442195 & -7.3 & -6.5 \\
\hline & 4 & Emetine & & 10219 & -7.9 & -5.6 \\
\hline & 5 & Psychotrine & & 65380 & -8.0 & -6.1 \\
\hline & 6 & Alangine & & 10851977 & -6.1 & -5.4 \\
\hline
\end{tabular}




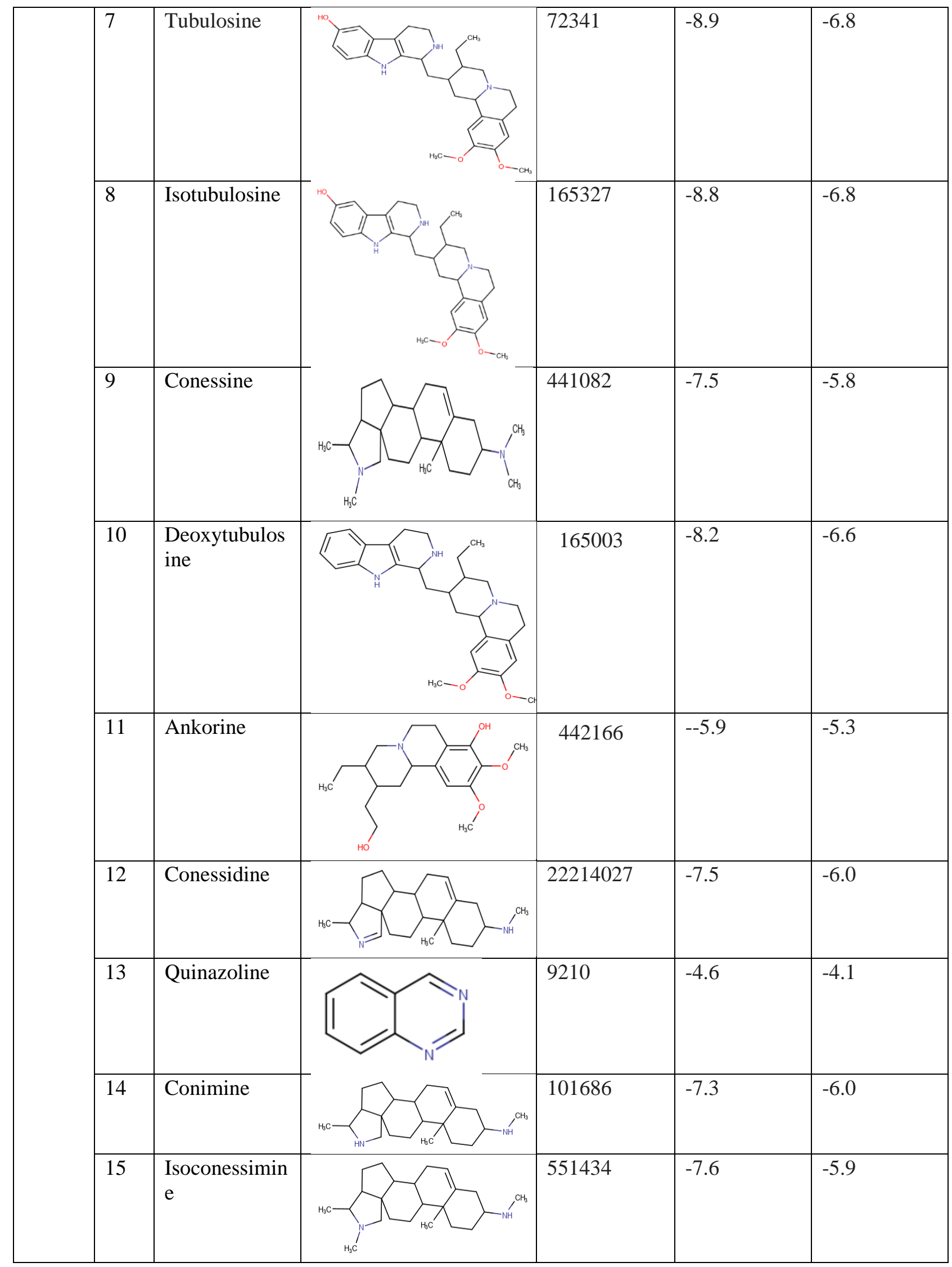




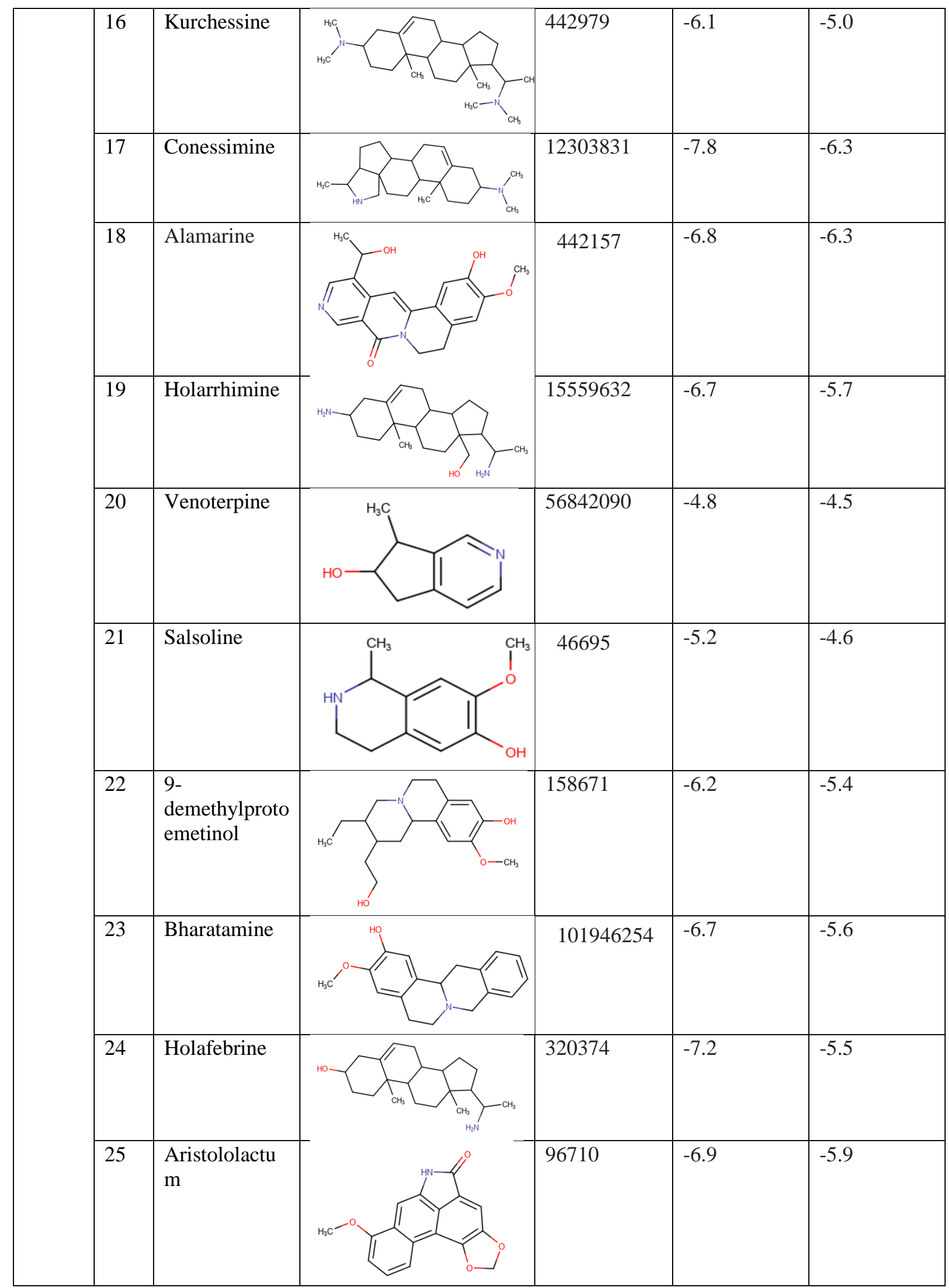




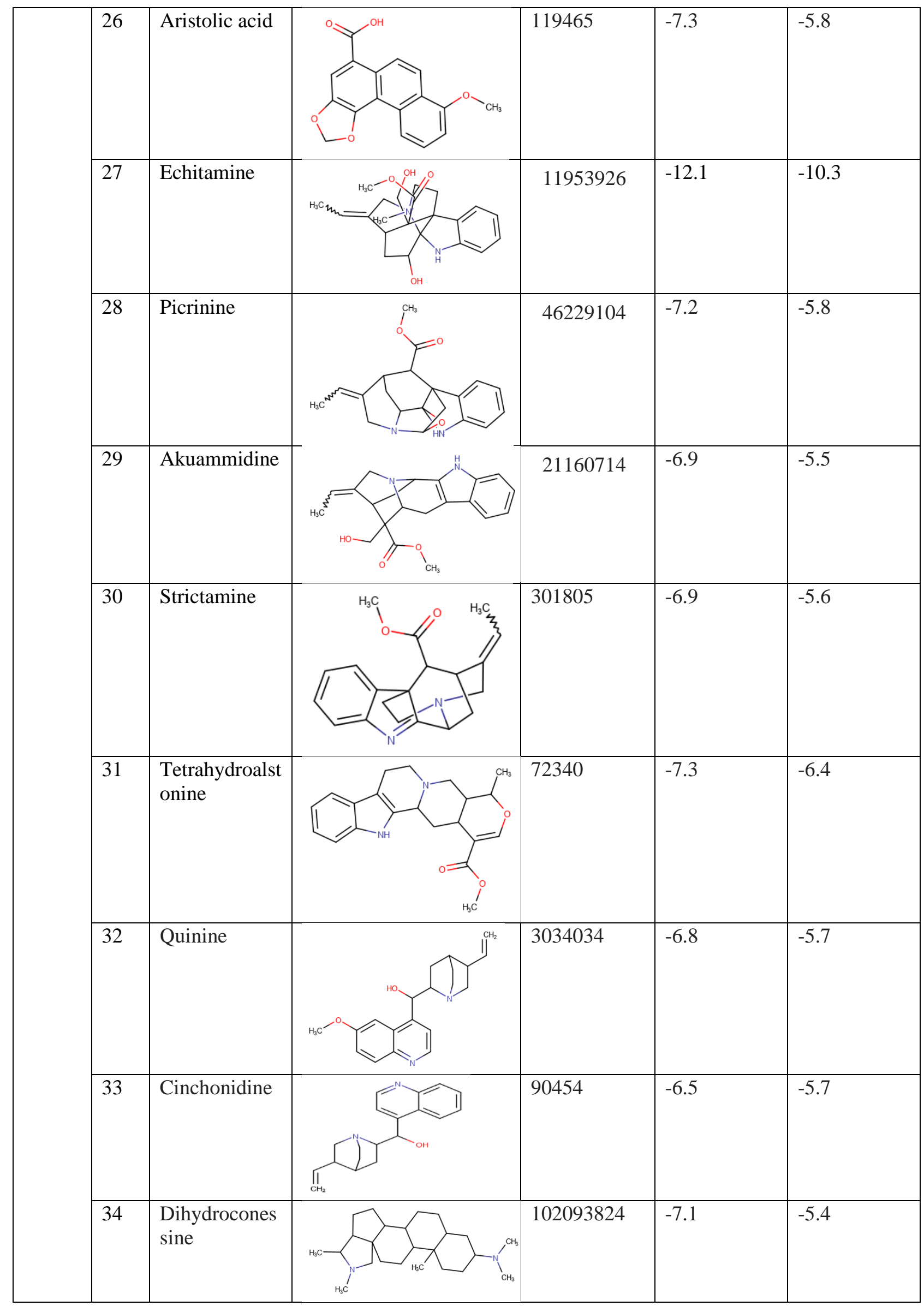




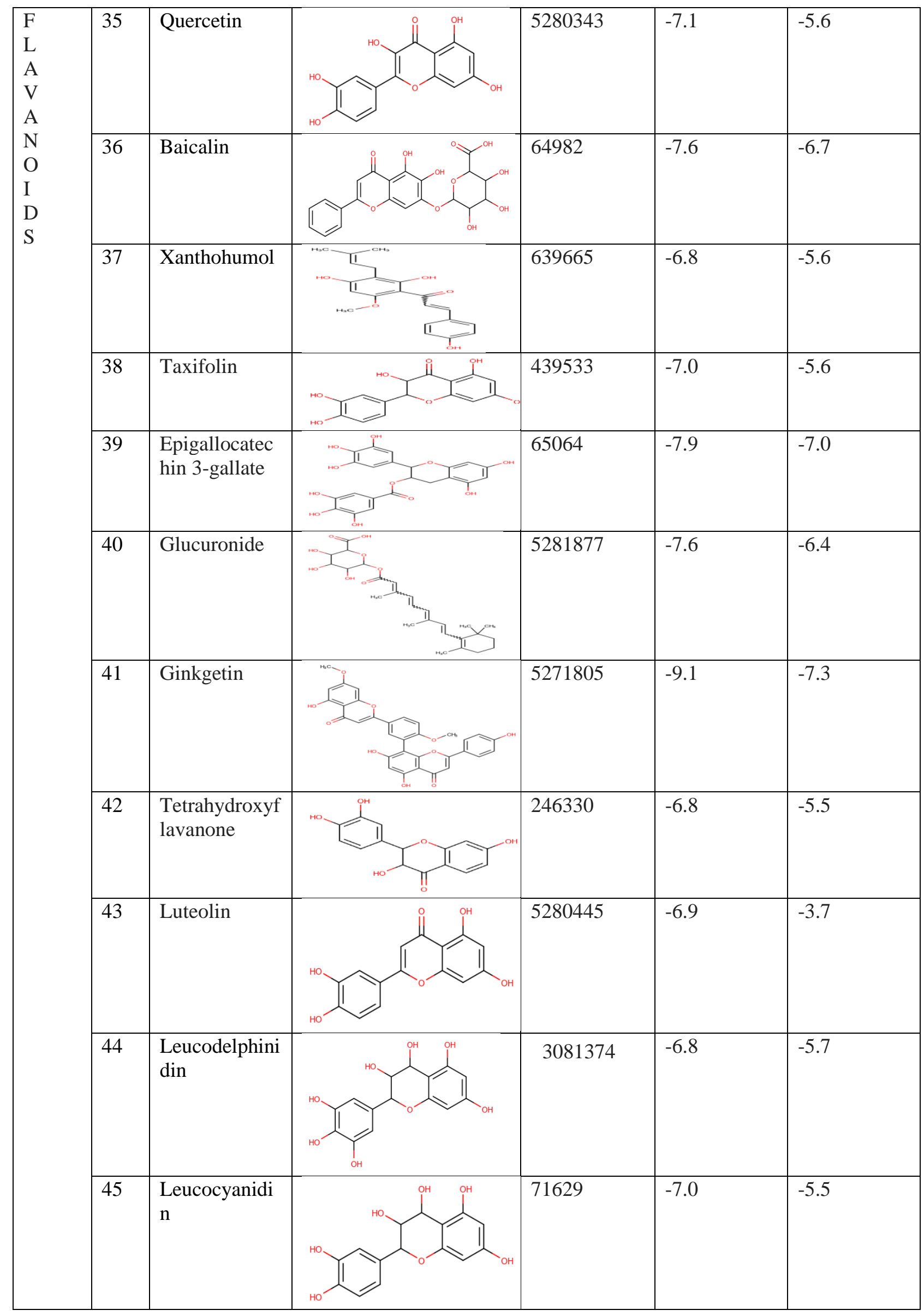




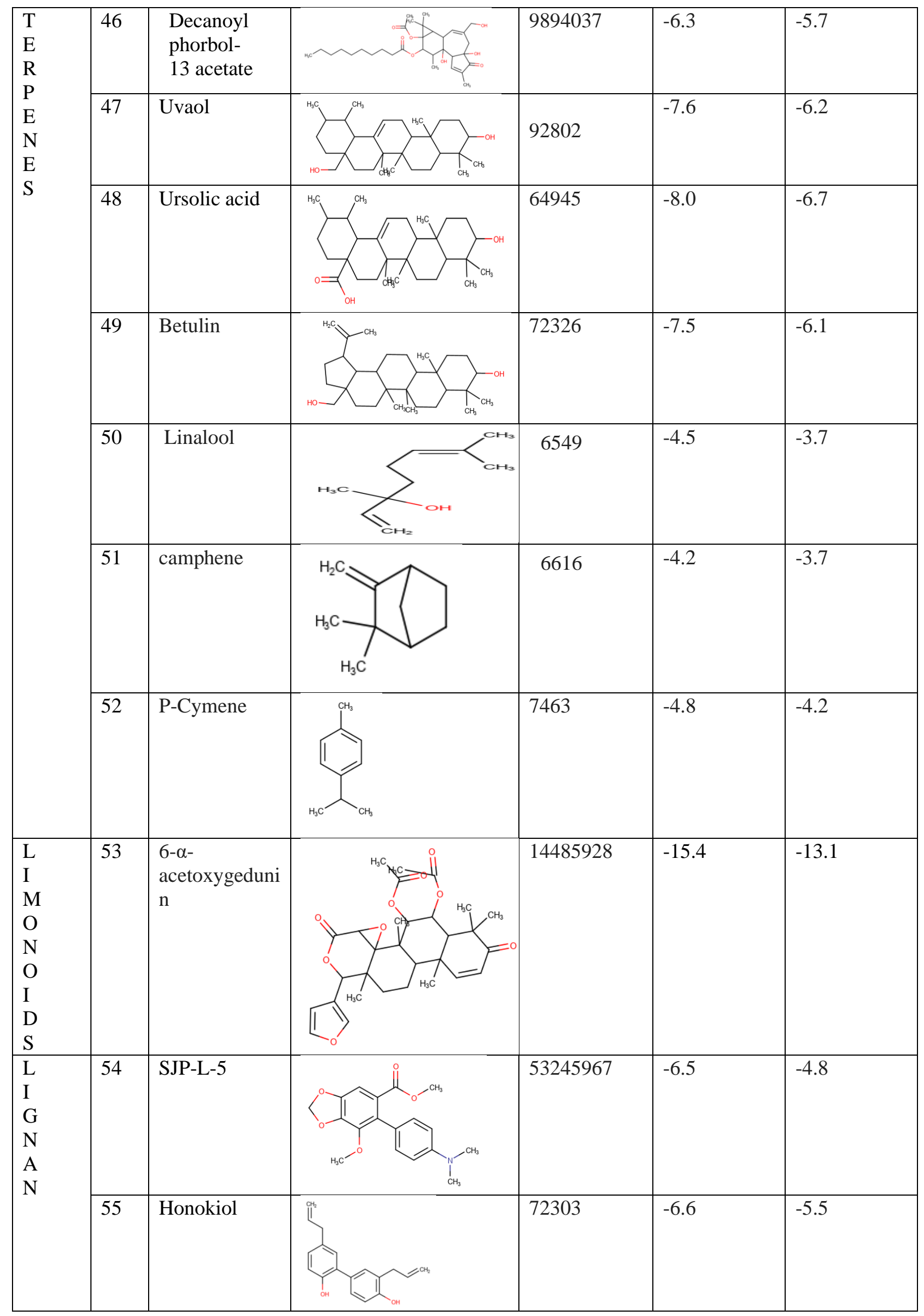




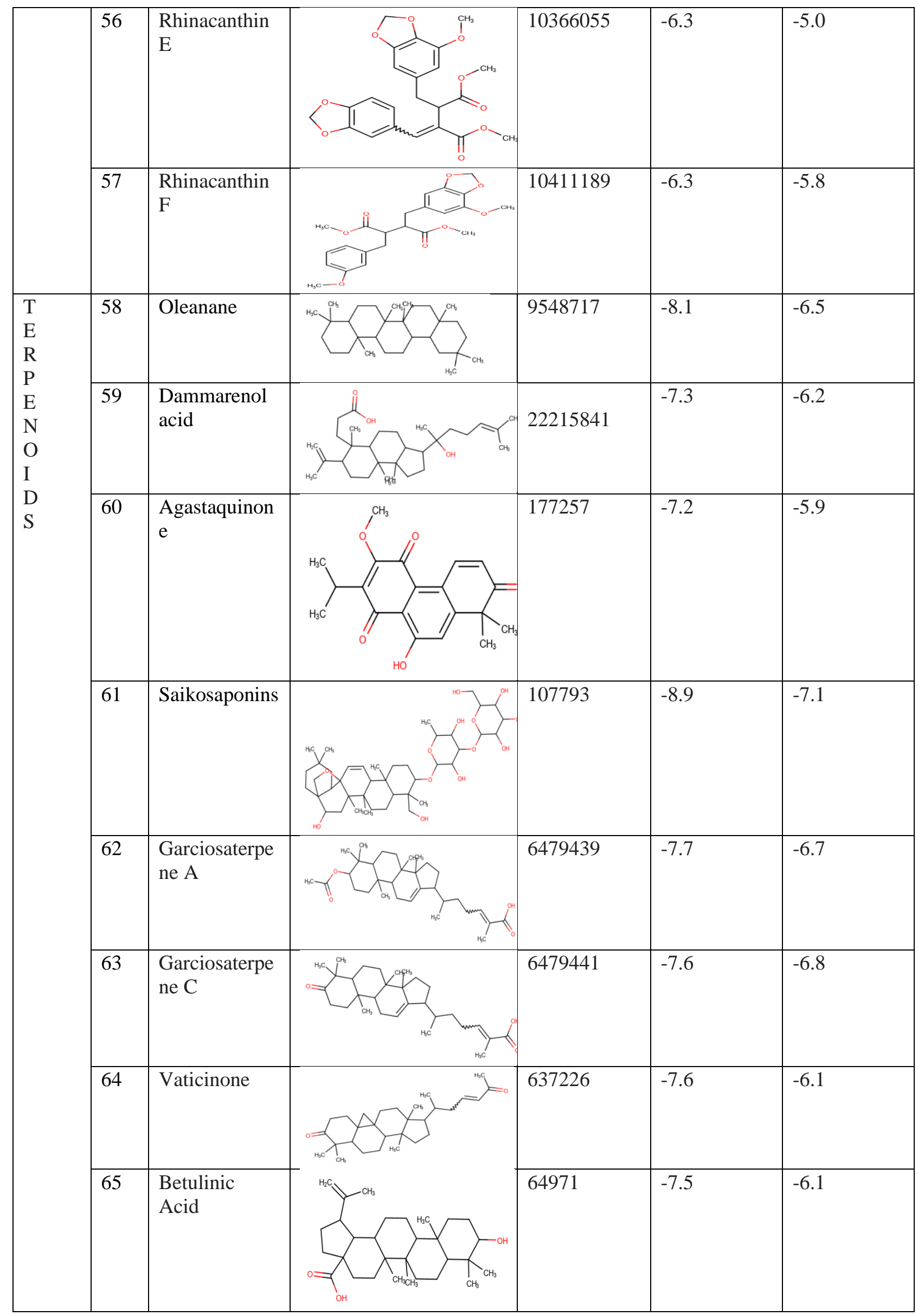




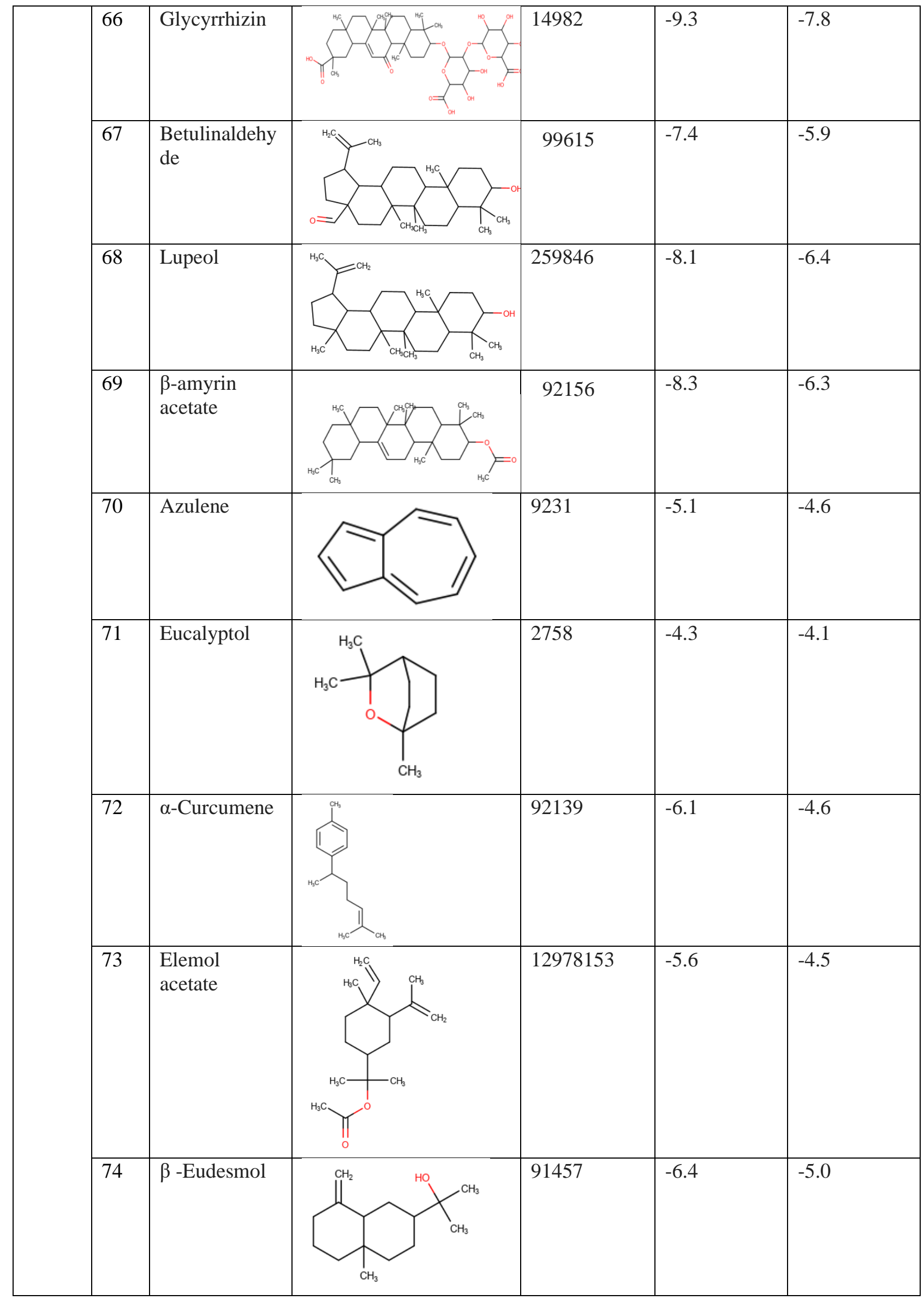




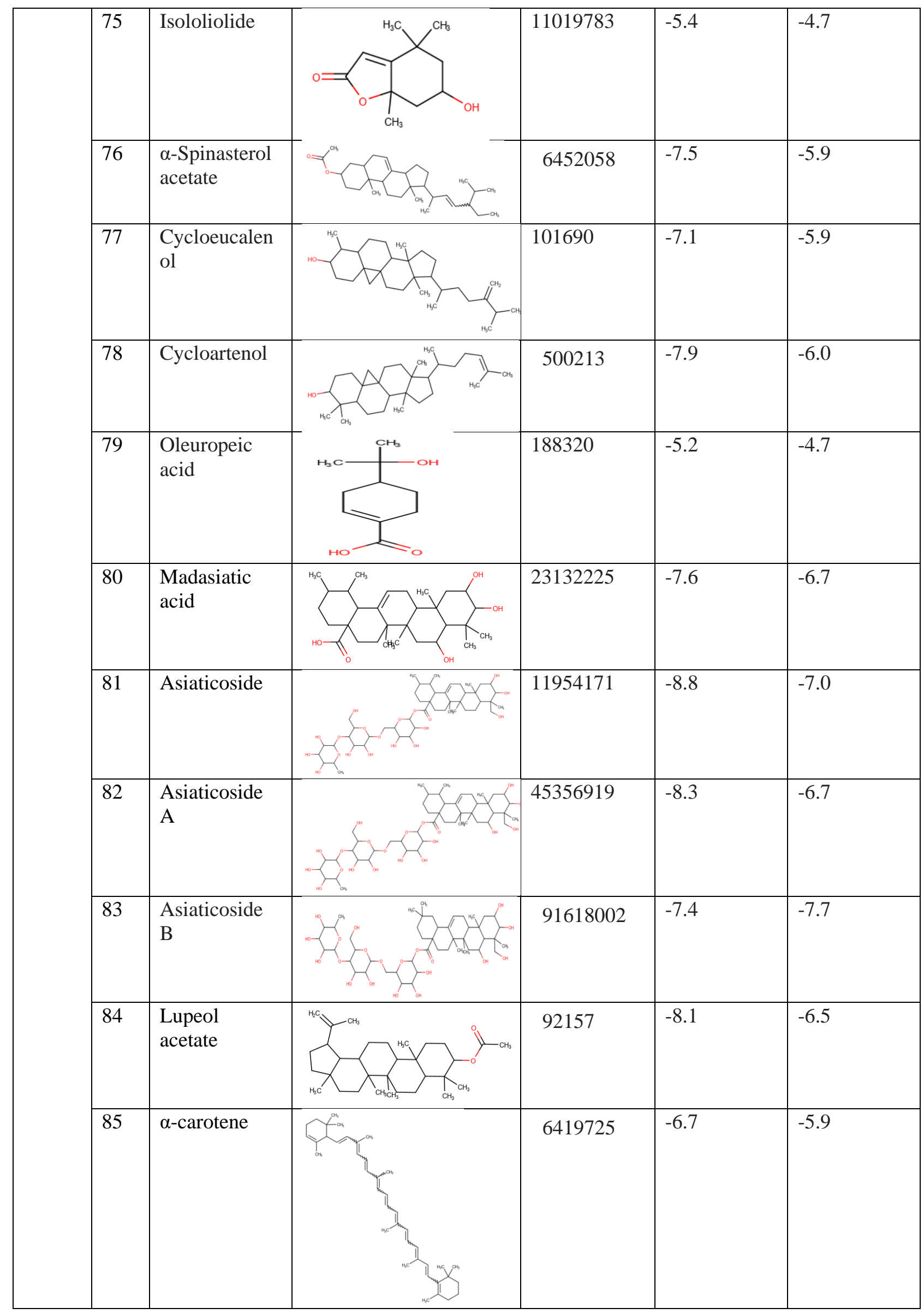




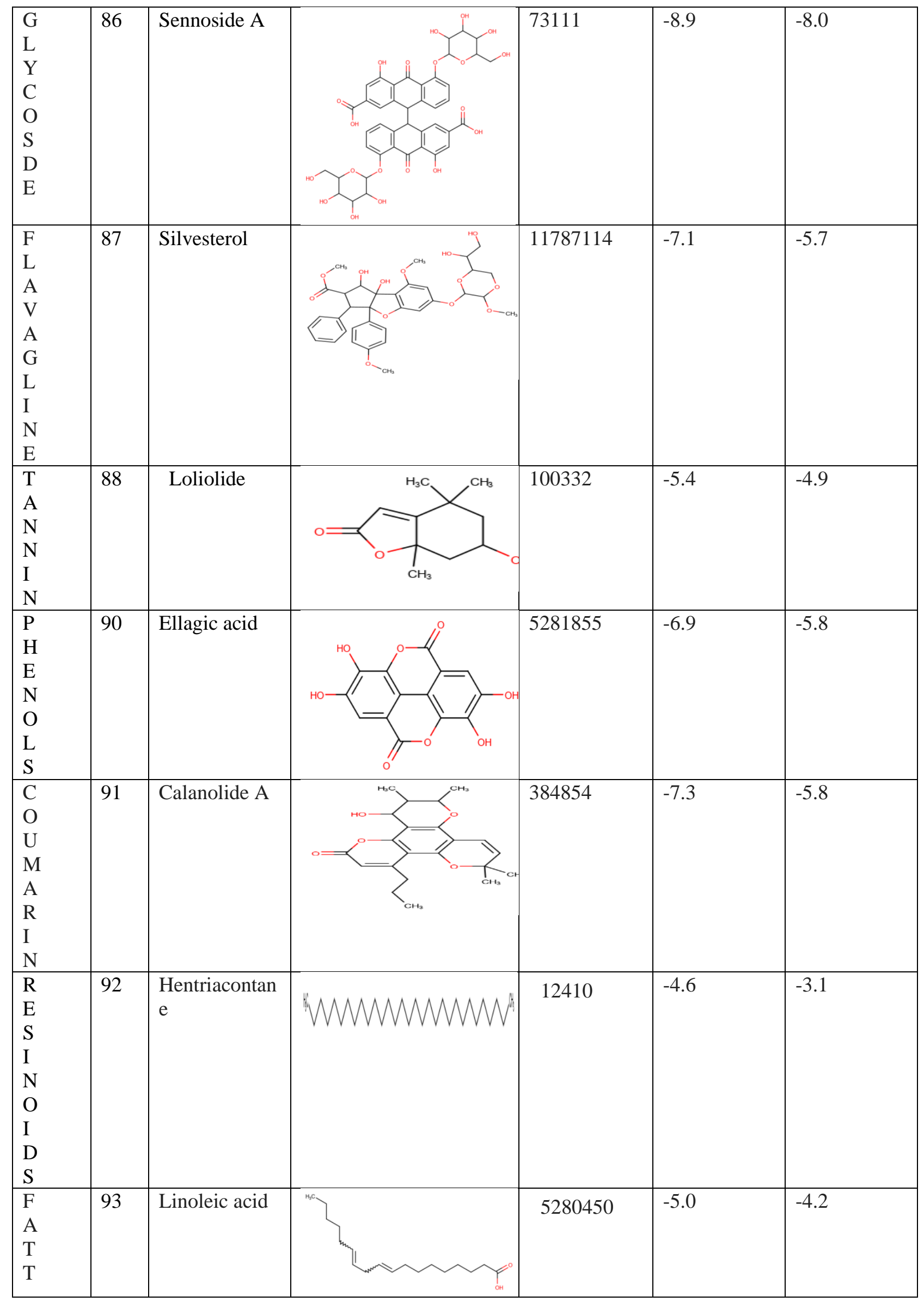




\begin{tabular}{|c|c|c|c|c|c|c|}
\hline \multirow{5}{*}{$\begin{array}{l}\text { Y } \\
\text { A } \\
\text { C } \\
\text { I } \\
\text { D } \\
\text { S }\end{array}$} & 94 & Oleic acid & MMMMi & 445639 & -4.4 & -3.8 \\
\hline & 95 & Palmitic acid & WW & 985 & -4.2 & -3.7 \\
\hline & 96 & Stearic acid & WWY & 5281 & -4.4 & -3.7 \\
\hline & 97 & Behenic acid & WMMMMY & 8215 & -4.7 & -3.7 \\
\hline & 98 & $\begin{array}{l}\text { Arachidic } \\
\text { acid }\end{array}$ & $M$ & 10467 & -4.1 & -3.7 \\
\hline \multirow{2}{*}{$\begin{array}{l}\mathrm{F} \\
\mathrm{L} \\
\mathrm{A} \\
\mathrm{V} \\
\mathrm{O} \\
\mathrm{N} \\
\mathrm{O} \\
\mathrm{L} \\
\mathrm{S}\end{array}$} & 99 & Chrysin & & 5281607 & -6.9 & -5.3 \\
\hline & 100 & Morin & & 5281670 & -6.8 & -5.5 \\
\hline \multirow[t]{3}{*}{$\begin{array}{l}\mathrm{P} \\
\mathrm{O} \\
\mathrm{L} \\
\mathrm{Y} \\
\mathrm{S} \\
\mathrm{A} \\
\mathrm{C} \\
\mathrm{C} \\
\mathrm{H} \\
\mathrm{A} \\
\mathrm{R} \\
\mathrm{I} \\
\mathrm{D} \\
\mathrm{E}\end{array}$} & 101 & $\begin{array}{l}\alpha-\mathrm{D}- \\
\text { Galacturonic } \\
\text { acid }\end{array}$ & & 445929 & -5.2 & -4.3 \\
\hline & 102 & Chloroquine & & 2719 & -5.4 & -4.6 \\
\hline & 103 & $\begin{array}{l}\text { Hydroxychlor } \\
\text { oquine }\end{array}$ & & 3652 & -5.7 & -4.7 \\
\hline
\end{tabular}




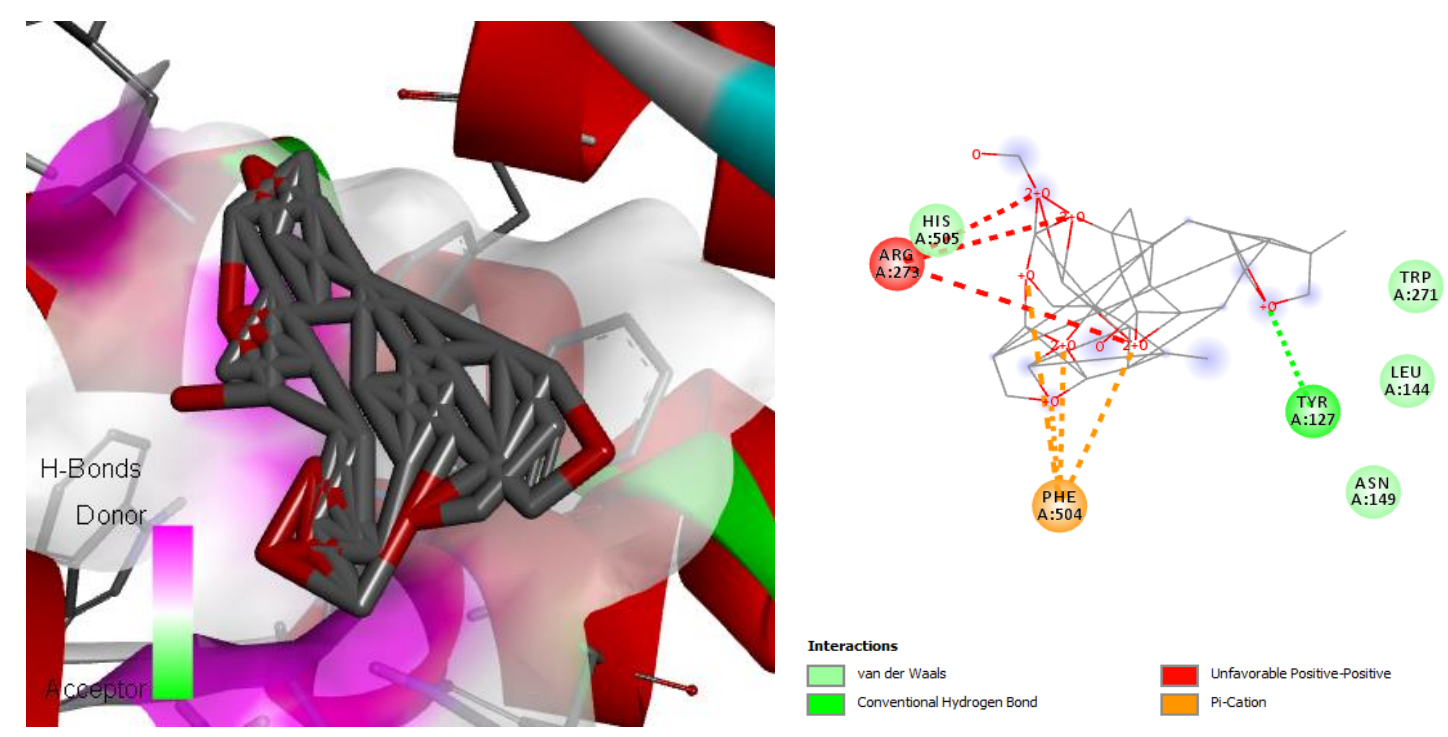

Figure 2. Binding interaction of 6-alpha-acetoxygedunin with 1R42
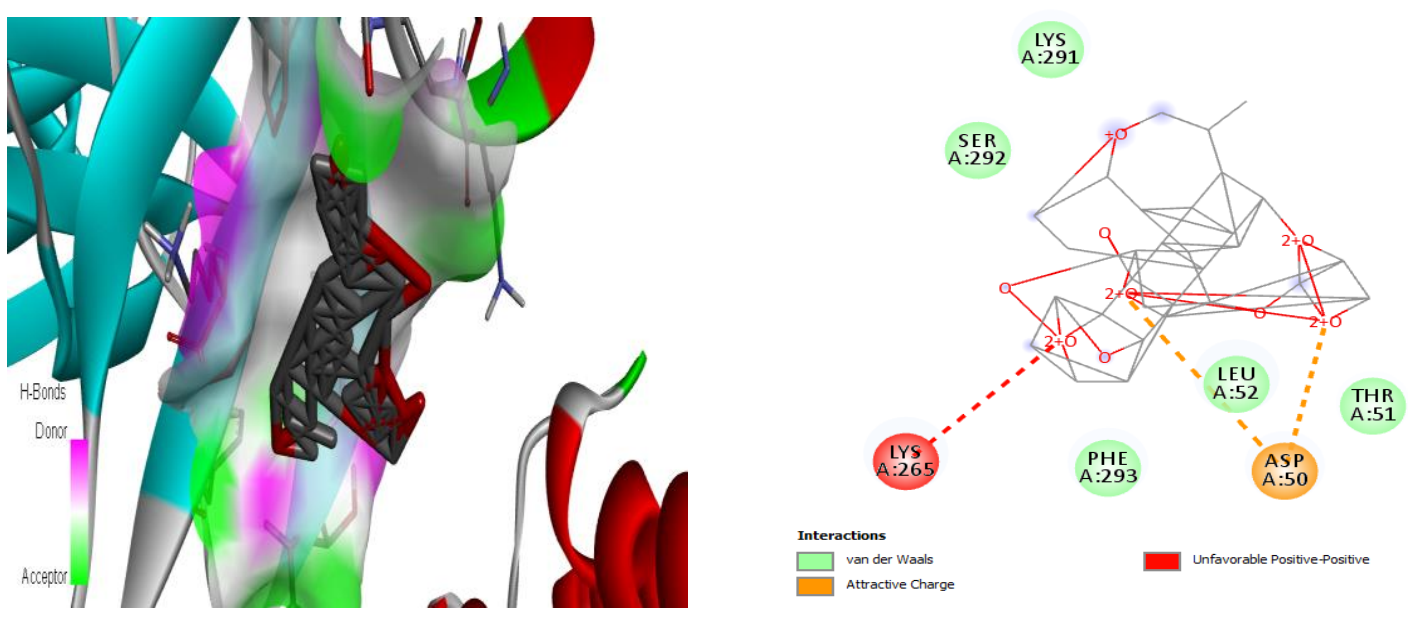

Figure 3. Binding interaction of 6-alpha-acetoxygedunin with 6CS2

\subsection{Assessment of ADMET}

The ADMET of the two selected candidate 6- $\alpha$-acetoxygedunin and echitamine were assessed (Table 3). The absorption of both the selected candidates was more than $98 \%$ indicating good absorption characteristics. The volume of distribution is low therefore drug stays more time in plasma. Both $6-\alpha-$ acetoxygedunin and echitamine are non-inhibitors of CYP isoenzymes (1 A2, 2C19, 2C9, 2D6, 3A4) except 6- $\alpha$-acetoxygedunin which is an inhibitor of 3A4. They are non-substrate of CYP2D6 and substrate of CYP3A4. Due to high lipophilicity both have low total renal clearance but these are nontransporters of renal OCT2 vital for drug disposition and renal excretion. They are non-mutagenic and non-carcinogenic as indicated by AMES toxicity test. 6 - $\alpha$-acetoxygedunin and echitamine have low LD50 score (3.529 and 3.302 respectively). Moreover, both these phytochemicals are very potent even in small dose. 
Table 3. ADMET/ TOX Properties of 6- $\alpha$-acetoxygedunin and echitamine.

\begin{tabular}{|c|c|c|c|c|}
\hline \multicolumn{3}{|c|}{ Properties } & $\begin{array}{c}6-\alpha- \\
\text { acetoxygedunin }\end{array}$ & Echitamine \\
\hline Absorption & \multicolumn{2}{|l|}{ IA (\%) } & 100 & 98.138 \\
\hline \multirow{2}{*}{ Distribution } & \multicolumn{2}{|l|}{ VDss(log L/kg) } & 0.115 & 0.789 \\
\hline & \multicolumn{2}{|l|}{ BBBP (Log BB) } & -1.073 & -0.212 \\
\hline \multirow{7}{*}{ Metabolism } & \multirow{5}{*}{ CYP Inhibitor(Y/N) } & $1 \mathrm{~A} 2$ & $\mathrm{~N}$ & $\mathrm{~N}$ \\
\hline & & $2 \mathrm{C} 19$ & $\mathrm{~N}$ & $\mathrm{~N}$ \\
\hline & & $2 \mathrm{C} 9$ & $\mathrm{~N}$ & $\mathrm{~N}$ \\
\hline & & 2D6 & $\mathrm{N}$ & $\mathrm{N}$ \\
\hline & & $3 \mathrm{~A} 4$ & $\mathrm{Y}$ & $\mathrm{N}$ \\
\hline & \multirow{2}{*}{ CYP Substrate(Y/N) } & 2D6 & $\mathrm{N}$ & $\mathrm{N}$ \\
\hline & & $3 \mathrm{~A} 4$ & $\mathrm{Y}$ & $\mathrm{Y}$ \\
\hline \multirow{2}{*}{ Excretion } & \multicolumn{2}{|c|}{ Total clearance $(\log \mathrm{ml} / \mathrm{min} / \mathrm{kg})$} & 0.003 & 0.882 \\
\hline & \multicolumn{2}{|c|}{$\operatorname{ROS}(\mathrm{Y} / \mathrm{N})$} & $\mathrm{N}$ & $\mathrm{N}$ \\
\hline \multirow{2}{*}{ Toxicity } & \multicolumn{2}{|l|}{$\operatorname{AMES}(\mathrm{Y} / \mathrm{N})$} & $\mathrm{N}$ & $\mathrm{N}$ \\
\hline & \multicolumn{2}{|l|}{ LD50 ( $\mathrm{mol} / \mathrm{kg})$} & 3.529 & 3.302 \\
\hline
\end{tabular}

IA: Intestinal Absorption; VDss: Volume of distribution in human; BBB: Blood Brain Barrier permeability; TC:Total clearance; ROS: Renal Organic CationTransporter 2 Substrate.

\section{CONCLUSION}

ACE-2 inhibition plays a vital role in treatment against COVID -19 by blocking SARS coronavirus spike protein mediated cell fusion. 6 - $\alpha$-acetoxygedunin and echitamine exhibits lowest docking score thus have highest binding interactions with ACE-2 protein. On the whole, we conclude that the two phytochemicals 6 - $\alpha$-acetoxygedunin and echitamine have desired qualities to be a potent inhibitor of COVID -19. Thus, it is worth to carry out further investigations involving in-vitro and in-vivo studies on these molecules.

\section{ACKNOWLEDGMENTS}

Authors are grateful to Dr.S.C.Si Dean SPS, for encouragements. Special thanks are due to honorable President, Prof. M.R. Nayak of the University for providing necessary facilities.

\section{CONFLICT OF INTERESTS}

Authors declare no conflict of interest.

\section{ABBREVIATIONS:}

COVID-19: Coronavirus disease 2019; ADMET: Absorption, distribution, metabolism, excretion and toxicity; ACE: Angiotensin converting enzyme; RAS: Renin angiotensin system; SARS-CoV-2: severe acute respiratory syndrome coronavirus 2; OCT-2: Organic cation transporter 2. 


\section{REFERENCES}

1. Mehta P, McAuley DF, Brown M, Sanchez E, Tattersall RS, Manson JJ. COVID-19: consider cytokine storm syndromes and immunosuppression. The Lancet. 2020;395(10229):1033-4.

2. Zhu N, Zhang D, Wang W, Li X, Yang B, Song J, Zhao X, Huang B, Shi W, Lu R, Niu P. A novel coronavirus from patients with pneumonia in China, 2019. New England Journal of Medicine. 2020 Jan 24.

3. Zhou Y, Hou Y, Shen J, Huang Y, Martin W, Cheng F. Network-based drug repurposing for novel coronavirus 2019-nCoV/SARS-CoV-2. Cell Discovery. 2020; 6:14.

4. Hoffmann M, Kleine-Weber H, Krüger N, Mueller MA, Drosten C, Pöhlmann S. The novel coronavirus 2019 (2019-nCoV) uses the SARS-coronavirus receptor ACE2 and the cellular protease TMPRSS2 for entry into target cells. BioRxiv. 2020 Jan 1.

5. Li SR, Tang ZJ, Li ZH, Liu X. Searching therapeutic strategy of new coronavirus pneumonia from angiotensin-converting enzyme 2: the target of COVID-19 and SARS-CoV. European Journal of Clinical Microbiology \& Infectious Diseases. 2020; 39(6):1021-1026.

6. Xu, X., Chen, P., Wang, J., Feng, J., Zhou, H., Li, X., Zhong, W., Hao, P. Evolution of the novel coronavirus from the ongoing Wuhan outbreak and modeling of its spike protein for risk of human transmission. Sci China Life Sci 2020; 63(3):457-460.

7. Kapoor R, Sharma B, Kanwar SS. Antiviral phytochemicals: an overview. Biochem. Physiol. 2017; 6: 220 .

8. Naithani R, Huma LC, Holland LE, Shukla D, McCormick DL, Mehta RG, Moriarty RM. Antiviral activity of phytochemicals: a comprehensive review. Mini reviews in medicinal chemistry. 2008; 8(11):1106-33.

9. Mothana RA, Mentel R, Reiss C, Lindequist U. Phytochemical screening and antiviral activity of some medicinal plants from the island Soqotra. Phytotherapy Research: An International Journal Devoted to Pharmacological and Toxicological Evaluation of Natural Product Derivatives. 2006; 20(4):298-302.

10. Saddala MS, Kiran JA, Rani UA. In Silico drug design and molecular docking studies of potent Inhibitors against Cathepsin-L (Ctsl) for SARS disease. J Res Development. 2016; 4(145):2.

11. Vriend G. WHAT IF: a molecular modeling and drug design program. Journal of molecular graphics. 1990;8(1):52-6.

12. Dundas J, Ouyang Z, Tseng J, Binkowski A, Turpaz Y, Liang J. CASTp: computed atlas of surface topography of proteins with structural and topographical mapping of functionally annotated residues. Nucleic acids research. 2006; 34:116-8.

13. Sharma T. Insilico docking approach to study the binding affinity of Isoflavones on the crystal structure of estrogen receptor alpha. 2017; 54(10):7-15.

14. Akabli T, Lamchouri F, Senhaji S, Toufik H. Molecular docking, ADME/Tox prediction, and in vitro study of the cell growth inhibitory activity of five $\beta$-carboline alkaloids. Structural Chemistry. 2019; 30(4):1495-504.

15. Singh RP, Dhanalakshmi S, Agarwal R. Phytochemicals as cell cycle modulators a less toxic approach in halting human cancers. Cell cycle. 2002; 1(3):155-60. 